\title{
Medullary carcinoma of the large intestine: A population based analysis
}

\author{
PRAGATHEESHWAR THIRUNAVUKARASU ${ }^{1 *}$, MAGESH SATHAIAH $^{1 *}$, SMIT SINGLA $^{2}$, SHYAM SUKUMAR $^{3}$, \\ ARIVARASAN KARUNAMURTHY ${ }^{4}$, KOTHAI DIVYA PRAGATHEESHWAR ${ }^{1}$, KENNETH K.W. LEE ${ }^{5}$, \\ HERBERT ZEH III ${ }^{1}$, KEVIN M. KANE ${ }^{6}$ and DAVID L. BARTLETT ${ }^{1}$ \\ ${ }^{1}$ UPMC Cancer Pavilion, University of Pittsburgh, Rm 415, 5150 Center Avenue, Pittsburgh, PA 15232; ${ }^{2}$ University of \\ Pennsylvania, Suite 233 JMB, 3620 Hamilton Walk, Philadelphia, PA 19104; ${ }^{3}$ Henry Ford Health System, \\ Detroit, MI 48202, USA; ${ }^{4}$ Department of Pathology, K E M Hospital, Mumbai 400012, India; \\ ${ }^{5}$ University of Pittsburgh, 3550 Terrace Street 497 Scaife Hall, Pittsburgh, PA 15261; \\ ${ }^{6}$ McKeesport, Building D, 1500 5th Avenue, Pittsburgh, PA 15132, USA
}

Received April 27, 2010; Accepted June 7, 2010

DOI: 10.3892/ijo_00000741

\begin{abstract}
Medullary carcinoma (MC) of the colorectum is a relatively new histological type of adenocarcinoma characterized by poor glandular differentiation and intraepithelial lymphocytic infiltrate. To date, there has been no epidemiological study of this rare tumor type, which has now been incorporated as a separate entity in the World Health Organization (WHO) classification of colorectal cancers. We used the population-based registries of the Surveillance, Epidemiology and End Results (SEER) database to identify all cases of colorectal MC between 1973 and 2006 and compared them to poorly and undifferentiated colonic adenocarcinomas (PDA and UDA, respectively). We observed that MCs were rare tumors, constituting approximately 5-8 cases for every 10,000 colon cancers diagnosed, with a mean annual incidence of $3.47( \pm 0.75)$ per 10 million population. Mean age at diagnosis was $69.3( \pm 12.5)$ years, with incidence increasing with age. MCs were twice as common in females, who presented at a later age, with a lower stage and a trend towards favorable prognosis. MCs were extremely rare among AfricanAmericans. MCs were most common in the proximal colon $(74 \%)$, where they present at a later age than the sigmoid colon. There were no cases reliably identified in the rectum
\end{abstract}

Correspondence to: Dr David L. Bartlett, UPMC Cancer Pavilion, University of Pittsburgh, Rm 415, 5150 Center Avenue, Pittsburgh, PA 15232, USA

E-mail: bartdl@upmc.edu

*Contributed equally

Key words: medullary carcinoma, medullary-type adenocarcinoma, medullary carcinoma of colon, poorly differentiated adenocarcinoma, undifferentiated adenocarcinoma, microsatellite instability, colon, adenocarcinoma or appendix. Serum carcinoembryonic antigen levels (CEA) were elevated prior to first course of treatment in $40 \%$ of the patients. MCs were more commonly poorly differentiated (72\%), with $22 \%$ being undifferentiated. MCs commonly presented with Stage II disease, with $10 \%$ presenting with metastases. Only one patient presented with $\mathrm{N} 2 \mathrm{~b}$ disease ( $>7$ positive nodes). Early outcome analyses showed that MCs have 1- and 2-year relative survival rates of 92.7 and $73.8 \%$ respectively. Although MCs showed a trend towards better early overall survival, undifferentiated MCs present more commonly with Stage III, with comparatively worse early outcomes.

\section{Introduction}

Medullary carcinoma (MC) of the colorectum is a distinct morphological type of minimally differentiated adenocarcinoma that has been increasingly recognized and studied in the last two decades (1). Formerly known as 'large cell adenocarcinoma with minimal differentiation', it is now being referred to as 'medullary' carcinoma, due to its organoid architecture that appeared to be similar to the pattern in developing embryonic organs (1). However, it is important not to use this term in colorectal literature with the same connotation as it is used in breast and thyroid, as the pathological and clinical attributes are different. MC has been included as a distinct histological type in the World Health Organization (WHO) classification of colorectal epithelial cancers, wherein it is described as being characterized by sheets of malignant cells with vesicular nuclei, and prominent nucleoli, along with prominent intraepithelial lymphocytic infiltrate (2). In essence, MCs are a solid variety of adenocarcinoma with very little glandular differentiation. Although they are morphologically similar to poorly differentiated adenocarcinoma (PDA) (Fig. 1) and undifferentiated adenocarcinoma (UDA), they tend to display a distinct clinical behavior, in that they are typically more common in older females, less likely to present with nodal involvement and generally bear a better prognosis (3). Another characteristic 
feature of MCs is its strong association with microsatellite instability (MSI) in up to $60 \%$ (3-6).

MCs have been reported to constitute only $0.03 \%$ of all surgical sporadic colorectal carcinoma specimens (3). This rarity combined with its recent recognition as a distinct entity has made it difficult to study the epidemiological features of this cancer, with no reliable population-based data to date. In this study, we have performed a descriptive analysis of the epidemiological features of medullary carcinoma using a reliable national database wherein histological types are coded using standardized coding systems. We have used PDA and UDA from the same database during the same time period to perform a comparative analysis, in order to understand their clinico-epidemiological behavior with respected to poorly and undifferentiated adenocarcinomas, of which they are a special subset.

\section{Materials and methods}

SEER database. The Surveillance, Epidemiology and End Results (SEER) database is a National Cancer Institute (NCI)based source of cancer data from several population-based registries in the USA, with a representative coverage of $26 \%$ of the US population (7). The registries in the SEER program include Alaska Native Tumor Registry, Arizona Indians, Los Angeles, San Francisco-Oakland, San Jose-Monterey, Greater California, Connecticut, Detroit, Atlanta, Rural Georgia, Hawaii, Iowa, Kentucky, Louisiana, New Jersey, New Mexico, Seattle-Puget Sound and Utah. There are several groupings of these registries with names identifiable by the number of registries in each grouping, such as SEER 9, SEER 11, SEER 17, etc., each with distinct and overlapping uses. For population-based statistics, SEER 9 (1973-2006) is used, whereas for other statistics such as survival, SEER 17 (the most extensive grouping) can be used. Please visit http:// seer.cancer.gov/registries/terms.html for more information.

We used ICD-0-3 histological code '8510/3: Medullary Carcinoma, NOS' to identify all cases of malignant medullary carcinoma $(n=74)$, arising from the colon and rectum between 1973 and 2006. As medullary carcinoma is a relatively newly described histopathological type in the colon, we chose to use only cases diagnosed after $2004(n=50)$, for most analyses. We used 11,093 cases of poorly differentiated adenocarcinomas (PDA) and 720 cases of undifferentiated adenocarcinomas (UDA) identified in the same database during the same time period for comparison. Only microscopically confirmed cases were included in this study. MCs identified in the database were further grouped into poorly differentiated (PD-MC) and undifferentiated (UD-MC) types, based on their histological grade. For cases diagnosed since 2004, SEER database provided accurate $\mathrm{T}, \mathrm{N}$ and $\mathrm{M}$ staging information from which we derived the most recent American Joint Committee on Cancer (AJCC, 7th edition) stage (8) using the 'extent of disease' and 'number of positive nodes' codes.

Statistical analyses. We used SEER*Stat software, a PC tool that derives simple population-based epidemiological calculations provided by the SEER program. The data were analyzed using SPSS software (SPSS 17, IBM, Chicago, IL). Survival analysis was performed using Kaplan-Meier curves
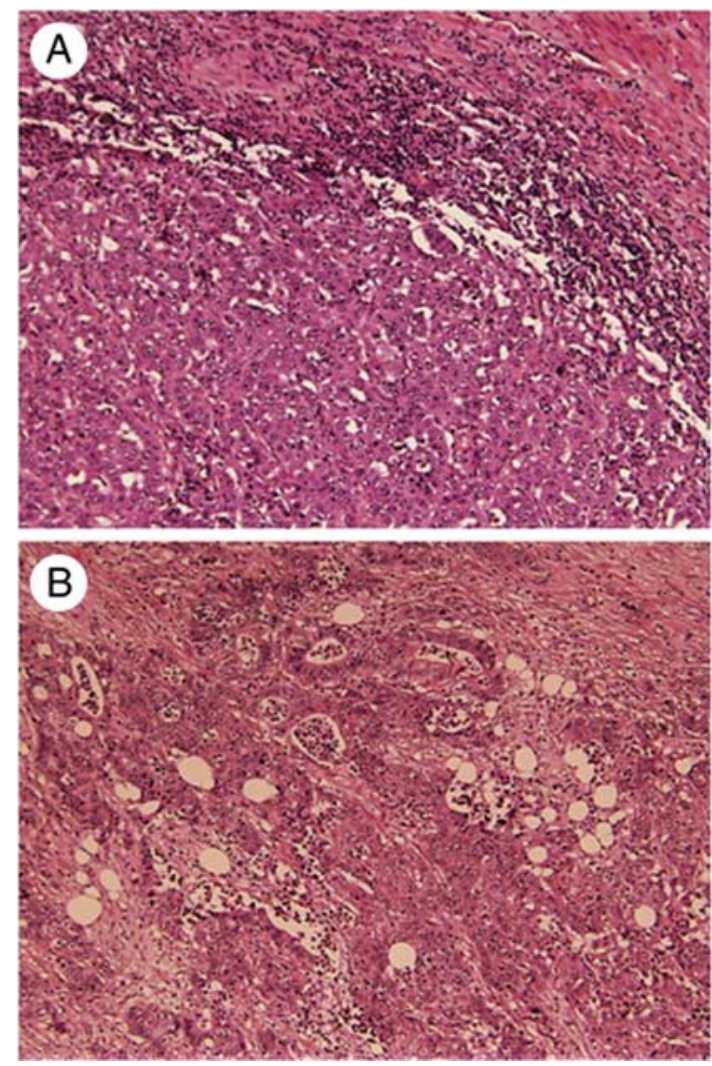

Figure 1. Histological appearance with hematoxylin and eosin stains of (A) medullary carcinoma (original magnification $\mathrm{x} 25$ ) and (B) poorly differentiated adenocarcinoma (original magnification $\mathrm{x} 40$ ). Reproduced with permission from Hum Pathol (Elsevier) (13).

for overall survival (OS) and statistical significance (p-value) was tested using log-rank (Mantel-Cox) test. Breslow's (generalized Wilcoxon) and Tarone-Ware tests were also used to confirm statistical significance in overall survival comparisons. Relative survival values were derived using the SEER*Stat software. An $\alpha$ value (p) of 0.05 to identify statistical significance was uniformly adopted for all statistical analyses. Mean values were expressed along with the standard deviation (SD) and statistical significance was tested using Student's t-test with two-tailed p-values. Standard error (SE) or 95\% confidence intervals $(\mathrm{CI})$ were provided for all median survival estimates.

\section{Results}

Patient characteristics and demographic data are shown in Table I.

Age at diagnosis. The mean age at diagnosis of $\mathrm{MC}$ of the large intestine was $69.3 \pm 12.5$ years. The mean age at diagnosis of males was $64.3 \pm 13.3$ years, which was much younger than the mean age of females, $72.1 \pm 11.2$ years (t-test, two tailed $\mathrm{p}=0.009$ ). MCs of the sigmoid colon tended to present earlier (mean age of $59.7 \pm 7.2$ years) than MCs in the rest of the colon (two-tailed $\mathrm{p}=0.05$ ). There was no significant difference in the mean age at diagnosis of poorly differentiated and undifferentiated types of MC. The mean ages at diagnosis of PDA and UDA were 69.2 \pm 14.2 years and 75.1 \pm 14.1 years 
Table I. Patient characteristics and demographics.

\begin{tabular}{|c|c|c|}
\hline Patient characteristic & No. $(n=50)$ & Percentage $(\%)$ \\
\hline \multicolumn{3}{|l|}{ Sex } \\
\hline Male & 16 & 32 \\
\hline Female & 34 & 68 \\
\hline \multicolumn{3}{|l|}{ Race } \\
\hline Caucasian & 44 & 88 \\
\hline $\mathrm{AI} / \mathrm{AK} / \mathrm{A} / \mathrm{PI}^{\mathrm{a}}$ & 6 & 12 \\
\hline African American & 0 & 0 \\
\hline \multicolumn{3}{|l|}{ SEER registry } \\
\hline California & 9 & 18 \\
\hline Connecticut & 6 & 12 \\
\hline Detroit (Metropolitan) & 10 & 20 \\
\hline Hawaii & 1 & 2 \\
\hline Iowa & 9 & 18 \\
\hline Kentucky & 7 & 14 \\
\hline Los Angeles & 3 & 6 \\
\hline Louisiana & 1 & 2 \\
\hline San Jose - Monterey & 2 & 4 \\
\hline Seattle (Puget Sound) & 2 & 4 \\
\hline \multicolumn{3}{|l|}{ Grade } \\
\hline Moderately differentiated & 2 & 4 \\
\hline Poorly differentiated & 36 & 72 \\
\hline Undifferentiated & 11 & 22 \\
\hline Unknown & 1 & 2 \\
\hline \multicolumn{3}{|l|}{ T stage ${ }^{b}$} \\
\hline $\mathrm{T} 1$ & 2 & 4 \\
\hline $\mathrm{T} 2$ & 8 & 16 \\
\hline $\mathrm{T} 3$ & 24 & 48 \\
\hline $\mathrm{T} 4 \mathrm{a}$ & 6 & 12 \\
\hline $\mathrm{T} 4 \mathrm{~b}$ & 10 & 20 \\
\hline \multicolumn{3}{|l|}{$\mathrm{N}$ stage } \\
\hline NO & 30 & 60 \\
\hline N1 & 12 & 24 \\
\hline $\mathrm{N} 2 \mathrm{a}$ & 7 & 14 \\
\hline $\mathrm{N} 2 \mathrm{~b}$ & 1 & 2 \\
\hline \multicolumn{3}{|l|}{ M stage } \\
\hline M0 & 45 & 90 \\
\hline M1 & 5 & 10 \\
\hline \multicolumn{3}{|l|}{ CEA level } \\
\hline Elevated & 10 & 20 \\
\hline Normal & 15 & 30 \\
\hline Not available & 25 & 50 \\
\hline
\end{tabular}

aAI, American Indian; AK, Alaska Native; A, Asian; PI, Pacific Islander. ${ }^{b} \mathrm{~N}$ stage, as per American Joint Committee on Cancer (AJCC), 7th edition staging. N2a, 4-6 nodes; N2b, $\geq 7$ nodes.

respectively, with no significant difference from $\mathrm{MC}$ although PDA also presented at a later age in females, than in males.

Sex and race distribution. There was a strong predilection for the female sex, with a male:female ratio of $1: 2.12$. This difference was steeper with PD-MC (1:2.27) than with UD-MC

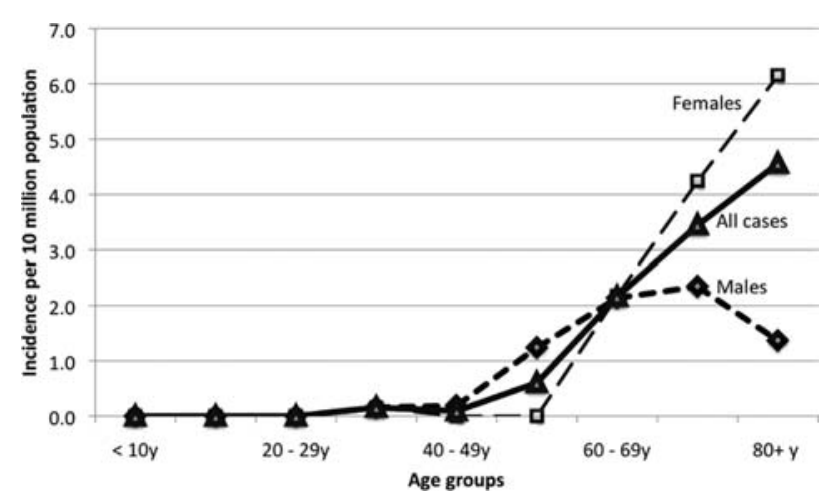

Figure 2. Incidence of medullary carcinoma by age.

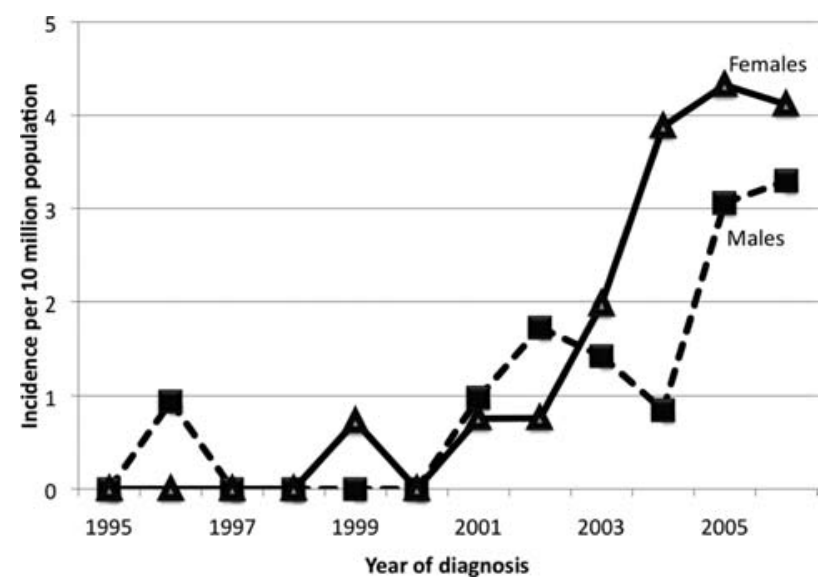

Figure 3. Temporal trends in incidence rates. The exponential increase in incidence since 2000 may be an artifact due to increasing awareness of medullary carcinoma as a separate histological entity.

(1:1.75). The sex ratios for PDA and UDA were $1: 1.15$ and $1: 1.42$, respectively. About $88 \%$ of MC patients were Caucasian and the rest were American Indian/Alaska Native/Pacific Islanders. There were no African-Americans identified in the database with MC since 2004, showing a negligible prevalence in this racial group. In contrast, $8.1 \%$ of PDA and $9.7 \%$ of UDA patients were African-Americans.

Incidence and temporal trends in incidence rates. The mean annual incidence of MC calculated using SEER 9 registry data from $2004-2006$ was $3.47 \pm 0.75$ per 10 million population. Age-adjusted analyses showed that the incidence of MC increased with age in a linear fashion. In males, the incidence peaked at the 8th decade, whereas in females it continued to increase (Fig. 2). There were no cases before the age of 30 years. When temporal trends in incidence rates were analyzed, there appeared to be an exponential rise since 2000 (Fig. 3). However, this may be an artifact due to the relatively recent categorization of $\mathrm{MC}$, which is the reason why we chose to use cases since 2004 for most analyses. The trends since 2004 suggest that females have a higher incidence rate than males. By calculating the total number of microscopically confirmed colon cancers diagnosed for the years 2004, 2005 and 2006 in SEER 17 registries, we estimated that MCs constituted approximately 5-8 cases for every 10,000 colon cancers diagnosed. 
Table II. Distribution of site of primary malignancy.

\begin{tabular}{lrcr}
\hline Primary site of malignancy & $\begin{array}{c}\mathrm{MC}(\%) \\
(\mathrm{n}=50)\end{array}$ & $\begin{array}{c}\text { PDA (\%) } \\
(\mathrm{n}=11,044)\end{array}$ & $\begin{array}{r}\text { UDA (\%) } \\
(\mathrm{n}=716)\end{array}$ \\
\hline Cecum & 36 & 21.75 & 25.70 \\
Appendix & 0 & 0.54 & 0.28 \\
Ascending colon & 38 & 18.98 & 22.63 \\
Hepatic flexure & 4 & 5.67 & 4.47 \\
Transverse colon & 12 & 8.30 & 8.52 \\
Splenic flexure & 2 & 2.92 & 2.79 \\
Descending colon & 2 & 4.0 & 2.51 \\
Sigmoid colon & 6 & 16.64 & 13.13 \\
Overlapping lesion of colon & 0 & 1.23 & 0.98 \\
Rectosigmoid junction & 0 & 6.96 & 5.87 \\
Rectum & 0 & 12.99 & 13.13 \\
\hline
\end{tabular}

MC, medullary carcinoma; PDA, poorly differentiated adenocarcinoma; UDA, undifferentiated adenocarcinoma. Only cases with known exact site of malignancy were included for analysis.

Site distribution. MCs were most commonly found in the cecum $(36 \%)$ and the ascending colon $(38 \%)$. The next most common sites were the transverse colon $(12 \%)$ and the sigmoid colon $(6 \%)$. Hepatic flexure, splenic flexure, descending colon and rectum were rare locations for MCs. There were no cases identified in the appendix. In contrast, significant proportions of PDA and UDA were found in all parts of the colon, with the least prevalent site being the appendix (Table II).

Tumor grade. For cases diagnosed since $2004(\mathrm{n}=50)$, the majority of MCs were poorly differentiated (72\%) or undifferentiated $(22 \%)$. Two patients $(4 \%)$ were moderately differentiated and 1 patient did not have any tumor grade available in the database. When all cases diagnosed since 1973 were analyzed, this trend was maintained, although tumor grade information was unavailable in $6 \%$.

Staging, extent of disease and treatment. The median tumor size was $7 \mathrm{~cm}$ in both males and females. MCs most commonly presented as a T3 tumor (48\%) and without nodal involvement $(60 \%)$, hence being mostly Stage IIA. The disease was meta-

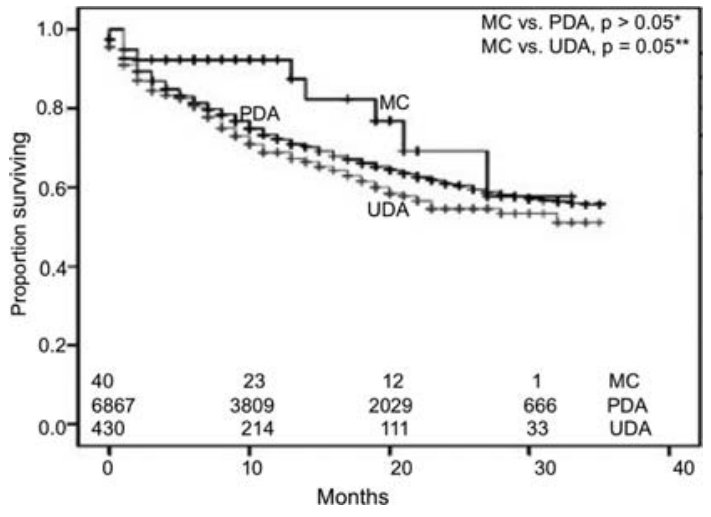

Figure 4. Kaplan-Meier curves for early outcomes by overall survival. *Not statistically significant despite better early outcomes, probably due to sample size limitations; generalized Wilcoxon, $\mathrm{p}=0.103$; Mantel-Cox (log-rank), $\mathrm{p}=0.204$; Tarone-Ware, $\mathrm{p}=0.126 .{ }^{* *}$ Generalized Wilcoxon, $\mathrm{p}=0.046$; MantelCox (log-rank), $\mathrm{p}=0.09$; Tarone-Ware, $\mathrm{p}=0.05$. The numbers at the bottom denote the number of patients at risk at each given time point in all groups compared. MC, medullary carcinoma; PDA, poorly differentiated adenocarcinoma; UDA, undifferentiated adenocarcinoma.

static in $10 \%$ of the patients at presentation. Men tended to present at later stages compared to women, with a higher prevalence of Stage III and IV disease. PD-MC subset had a similar pattern of stage distribution, whereas more than half of the patients with UD-MC presented with nodal involvement (Stage III). In comparison, both PDA and UDA followed patterns similar to UD-MC, in that they more commonly presented with Stage III disease (Table III). All patients diagnosed since 2004 underwent surgical treatment.

CEA levels. SEER began coding the status of serum CEA levels prior to the first course of treatment for patients diagnosed since 2004. CEA levels were coded using the Collaborative Staging criteria (9) with normal reference values being $<2.5 \mathrm{ng} / \mathrm{ml}$ (SI: $<2.5 \mu \mathrm{g} / \mathrm{l})$ for non-smokers and $<5 \mathrm{ng} / \mathrm{ml}(\mathrm{SI}:<5 \mu \mathrm{g} / \mathrm{l})$ for smokers when physician-/lab-aided interpretation is available. Only cases that had CEA levels available were used for analyses. CEA levels were elevated in $40 \%$ of MCs. A higher proportion of PDA and UDA (49.1 and 50.5\% respectively) patients had elevated CEA levels.

Risk of other malignancies in patients with MC. Ten patients (20\%) with MC developed another primary malignancy at

Table III. Stage distribution.

\begin{tabular}{lccccc}
\hline AJCC Stage $^{\mathrm{a}}$ & $\mathrm{MC}(\%)(\mathrm{n}=50)$ & $\mathrm{PD}-\mathrm{MC}(\%)(\mathrm{n}=36)$ & $\mathrm{UD}-\mathrm{MC}(\%)(\mathrm{n}=11)$ & PDA $(\%)(\mathrm{n}=10,878)$ & UDA $(\%)(\mathrm{n}=694)$ \\
\hline I & 12.0 & 13.89 & 0 & 8.81 & 9.80 \\
II & 44.0 & 47.22 & 45.45 & 28.06 & 27.38 \\
III & 34.0 & 25.0 & 54.55 & 40.10 & 38.90 \\
IV & 10.0 & 13.89 & 0 & 22.98 & 23.92 \\
\hline
\end{tabular}

MC, medullary carcinoma; PD-MC, poorly differentiated medullary carcinoma; UD-MC, undifferentiated medullary carcinoma; PDA, poorly differentiated adenocarcinoma; UDA, undifferentiated adenocarcinoma. ${ }^{a}$ American Joint Committee on Cancer Staging System, 7th edition. All patients with in situ cancer and unknown staging were excluded from the analysis. 


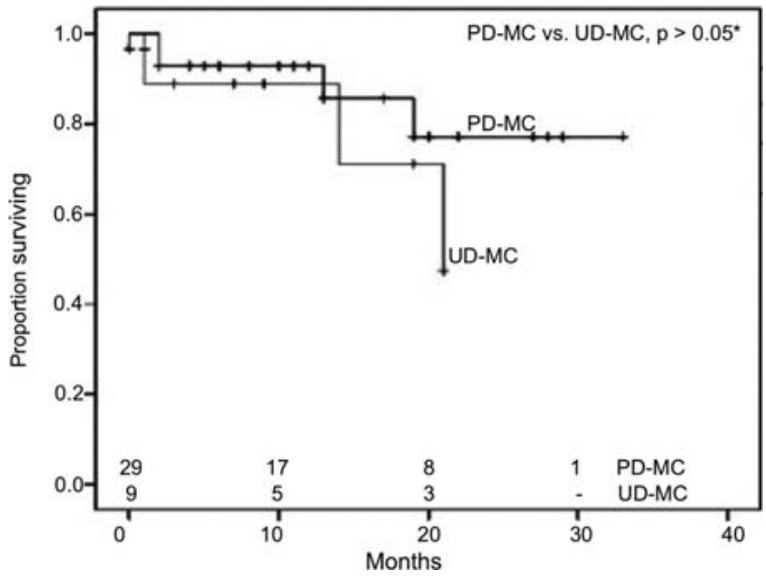

Figure 5. Kaplan-Meier curves for early outcomes by overall survival of medullary carcinomas by histological grade. *Not statistically significant despite better early outcomes probably due to sample size limitations; generalized Wilcoxon, $\mathrm{p}=0.546$; Mantel-Cox (log-rank), $\mathrm{p}=0.365$; TaroneWare, $\mathrm{p}=0.458$. The numbers at the bottom denote the number of patients at risk at each given time-point in all groups compared. PD-MC, poorly differentiated medullary carcinoma; UD-MC, undifferentiated medullary carcinoma.

some point during their lifetimes. In 6 of these patients, colonic MC was the first malignant primary. In these 6 patients, the other sites of primary were bladder (transitional cell carcinoma), prostate (adenocarcinoma), transverse and ascending colon (adenocarcinoma), duodenum (adenocarcinoma) and skin (lentigo maligna).

Survival analysis. We performed survival analyses using Kaplan-Meier method to look for differences in early overall survival (OS) outcomes between the two types of adenocarcinoma and poorly and undifferentiated MCs, after exclusion of patients who had other primary malignancies (Fig. 4). MCs appeared to have better survival outcomes than UDA $(n=430)$, reaching significance by generalized Wilcoxon $(\mathrm{p}=0.046)$ and

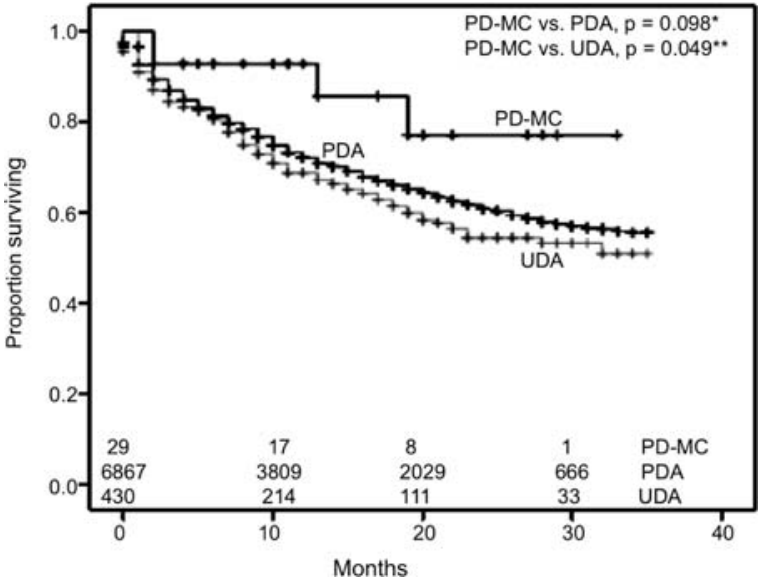

Figure 6. Kaplan-Meier curves for early outcomes by overall survival. *Not statistically significant despite better early outcomes probably due to sample size limitations; generalized Wilcoxon, $\mathrm{p}=0.105$; Mantel-Cox (log-rank), $\mathrm{p}=0.098$; Tarone-Ware, $\mathrm{p}=0.097 .{ }^{* *}$ Generalized Wilcoxon, $\mathrm{p}=0.058$; MantelCox $(\log$-rank $), p=0.049$; Tarone-Ware, $\mathrm{p}=0.049$. The numbers at the bottom denote the number of patients at risk at each given time-point in all groups compared. PD-MC, poorly differentiated medullary carcinoma; PDA, poorly differentiated adenocarcinoma; UDA, undifferentiated adenocarcinoma.

Tarone-Ware $(\mathrm{p}=0.05)$ tests, although statistical significance was close but not reached by Mantel-Cox (log-rank) test. There were no statistically significant differences in OS between MCs and PDA, although there appeared to be more favorable survival for MCs during the first 20 months. When PD-MC and UD-MC were compared, PD-MC appeared to have a favorable survival, although statistical significance was not reached for early outcome analyses (Fig. 5). PD-MC subset of patients showed a trend towards better OS than PDA and UDA (Fig. 6). We also derived relative survival (excluding other causes of death) from the SEER*Stat software's survival session by Kaplan-Meier methods for all those patients who were actively followed after exclusion of patients based on

Table IV. Relative 1- and 2-year survival rates.

Cumulative CI

Survival rate

Histological type

Relative survival

SE $(\%)$

Lower (\%)

Upper (\%) rate $^{\mathrm{a}}(\%)$

\begin{tabular}{llrrrr}
\hline 1 -year & & & & & \\
& MC & 92.70 & 4.90 & 73.20 & 93.20 \\
& PDA & 70.50 & 0.60 & 72.50 & 74.80 \\
2-year & 69.90 & 2.40 & 64.70 & 74.60 \\
& & & & \\
& UDA & 73.80 & 10.40 & 44.50 & 89.20 \\
& MC & 58.40 & 0.80 & 62.40 & 65.30 \\
& PDA & 53.10 & 3.30 & 51.40 & 64.30 \\
\hline
\end{tabular}

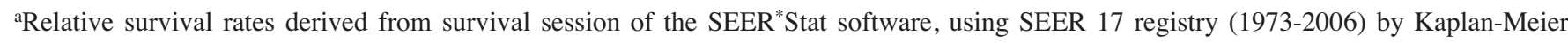
methods (with no adjustment to heterogeneity), with adjustments made when cumulative relative survival rates are higher than the prior period. MC, medullary carcinoma $(n=43)$; PDA, poorly differentiated adenocarcinoma $(n=7,497)$; UDA, undifferentiated adenocarcinoma $(\mathrm{n}=453)$; SE, standard error; CI, confidence intervals $(95 \%)$. 
multiple primary tumors, autopsy/death certificate only reports and patients not alive with no survival time mentioned. The results are shown in Table IV.

\section{Discussion}

Medullary carcinoma of the colon, being a relatively recent addition to the histological types of colorectal epithelial cancers, has been dealt with in only a handful of studies that have primarily dealt with its pathological aspects. As early as 1977, Gibbs reported a small series of undifferentiated adenocarcinomas with a tendency to grow to larger sizes before producing symptoms, but had a favorable prognosis (10). In 1997, Ruschoff et al reported a series of poorly differentiated non-glandular colorectal adenocarcinomas, most of which exhibited an expansive growth pattern and significant peritumor lymphoid infiltrate, resembling solid or medullary carcinomas of the stomach (11). They also noted that they had high MSI and generally showed good prognosis. In 1999, Jessurun et al described a series of 11 cases of colonic adenocarcinoma that displayed a predominantly solid, non-glandular component and at the same time were negative for neuroendocrine markers (12). All the patients were women with tumors in the cecum or proximal colon. Authors concluded that this non-glandular adenocarcinoma should be distinguished from the other more aggressive non-glandular adenocarcinomas. Lanza et al identified 45 MCs among 1,265 surgical specimens over a period of 10 years (3). They observed that these tumors were typically diploid, p53-negative, demonstrated widespread MSI, and had a favorable prognosis compared to well differentiated and poorly differentiated adenocarcinomas. Following this, a few other studies confirmed the high frequency of MSI, loss of p53 and CDX2 expression and favorable prognosis of MCs compared to PDA and neuroendocrine tumors of the colon $(1,4,5)$. A recent interesting observation was made by Winn et al (13), who noted that although MCs and UDA may be difficult to differentiate by light microscopy, MCs frequently stained positive for MUC-1, MUC-2 and TFF-3 indicating that MCs still retain intestinal differentiation to some degree. They also showed that they can be differentiated from other undifferentiated carcinomas by strong calretinin staining and loss of MLH-1 and CDX2 staining.

Our study, which aims to describe the clinical and epidemiological characteristics using a large population-based database, confirms some of the observations made in these earlier studies while making new observations as well. Our study shows that medullary carcinoma is probably much less common than suggested by Lanza et al (3) or other studies, mainly due to the lack of uniform criteria for defining this histological type in the other studies. For example, Lanza et al used the criteria of $>70 \%$ solid component and lack of nuclear pleomorphism as the criteria for MC, with tumor samples showing varying amounts of glandular differentiation. Our study used the ICD-O-3 coding mentioned in the WHO classification, which identifies medullary carcinomas as tumors with sheets of malignant cells with abundant pink cytoplasm, prominent nucleoli, vesicular nuclei and intraepithelial lymphocytic infiltrate (2) without any specific mention of the percentage of the differentiated or solid component.
Our study shows a disproportionate and exponential rise in the number of MCs diagnosed after 2000. However, this may be the result of MCs having been recognized as a separate entity only in the last decade, prior to which they might have simply been coded as UDA or PDA or even as neuroendocrine tumors, given their resemblance to the latter (1). In order to avoid this bias, we used only cases diagnosed since 2004, which is when it appeared on temporal incidence trend analysis that MCs were regularly identified to some extent. The limitation of such a decision is that only short-term survival outcomes can be reliably derived, given the fact that SEER database information was updated only until 2006. Our study however recognizes two classes of MCs based on their extent of differentiation. As expected, PD-MCs appear to have a more favorable prognosis than UD-MCs. The worse outcome of UD-MCs is probably the reason for the lack of significant differences in early survival outcomes between all MCs and PDA. Although most of our analyses show a visible difference in early survival, the rare nature of the tumor possibility deters statistical significance to be reached.

In conclusion, medullary carcinomas of the large intestine are a rare subset of minimally differentiated adenocarcinomas, which are more common in women and extremely rare in African Americans. They are more common in the proximal colon, with almost no occurrences in the rectum, pointing cues to possible role of site-specific variations such as colonic flora, intraluminal nutrient content and composition. Sigmoid MCs tend to present earlier than MCs at other colonic sites. MCs commonly present in Stage II and III, although presentation with $\mathrm{N} 2 \mathrm{~b}$ status (which is associated with significantly poor survival) is rare (8). Men tend to present at later stages, contributing to a trend in overall poor survival. A subset of MCs that are undifferentiated, fare worse than their poorly differentiated counterparts. The favorable prognosis of MCs in general is probably because of the fact that they still do retain some intestinal differentiation and coexisting lymphoid infiltration, indicating host reaction against tumor. However, such a conclusion would require further studies in the future including immunohistochemical analysis with regard to the specific subsets of MCs. Finally, the authors think that medullary carcinoma of the colon may be increasingly identified hereafter, possibly revealing new epidemiological patterns identifiable in the future, generating a need for long-term follow-up studies using similar large population-based databases.

\section{References}

1. Wick MR, Vitsky JL, Ritter JH, Swanson PE and Mills SE: Sporadic medullary carcinoma of the colon: a clinicopathologic comparison with nonhereditary poorly differentiated enterictype adenocarcinoma and neuroendocrine colorectal carcinoma. Am J Clin Pathol 123: 56-65, 2005.

2. Hamilton SR and Aaltonen LA (eds.): Pathology and genetics of tumors of the digestive system. In: World Health Organization Classification of Tumors. IARC press, Lyon, 2000.

3. Lanza G, Gafa R, Matteuzzi M and Santini A: Medullary-type poorly differentiated adenocarcinoma of the large bowel: a distinct clinicopathologic entity characterized by microsatellite instability and improved survival. J Clin Oncol 17: 2429-2438, 1999.

4. Alexander J, Watanabe T, Wu TT, Rashid A, Li S and Hamilton SR: Histopathological identification of colon cancer with microsatellite instability. Am J Pathol 158: 527-535, 2001. 
5. Hinoi T, Tani M, Lucas PC, et al: Loss of CDX2 expression and microsatellite instability are prominent features of large cell minimally differentiated carcinomas of the colon. Am J Pathol 159: 2239-2248, 2001

6. Kim H, Jen J, Vogelstein B and Hamilton SR: Clinical and pathological characteristics of sporadic colorectal carcinomas with DNA replication errors in microsatellite sequences. Am J Pathol 145: 148-156, 1994

7. Yu JB, Gross CP, Wilson LD and Smith BD: NCI SEER public-use data: applications and limitations in oncology research. Oncology 23: 288-295, 2009.

8. Gunderson LL, Jessup JM, Sargent DJ, Greene FL and Stewart AK: Revised tn categorization for colon cancer based on national survival outcomes data. J Clin Oncol 28: 264-271, 2010 .
9. Collaborative stage data collection system coding instructions. www.cancerstaging.org

10. Gibbs NM: Undifferentiated carcinoma of the large intestine. Histopathology 1: 77-84, 1977.

11. Ruschoff J, Dietmaier W, Luttges J, et al: Poorly differentiated colonic adenocarcinoma, medullary type: clinical, phenotypic, and molecular characteristics. Am J Pathol 150: 1815-1825, 1997.

12. Jessurun J, Romero-Guadarrama M and Manivel JC: Medullary adenocarcinoma of the colon: clinicopathologic study of 11 cases. Hum Pathol 30: 843-848, 1999.

13. Winn B, Tavares R, Fanion J, et al: Differentiating the undifferentiated: immunohistochemical profile of medullary carcinoma of the colon with an emphasis on intestinal differentiation. Hum Pathol 40: 398-404, 2009. 